\title{
BMJ Open Diagnosed diabetes and premature death among middle-aged Japanese: results from a large-scale population- based cohort study in Japan (JPHC study)
}

Masayuki Kato, ${ }^{1,2}$ Mitsuhiko Noda, ${ }^{1}$ Tetsuya Mizoue, ${ }^{3}$ Atsushi Goto, ${ }^{1,4}$ Yoshihiko Takahashi, ${ }^{5}$ Yumi Matsushita, ${ }^{6}$ Akiko Nanri, ${ }^{3}$ Hiroyasu Iso, ${ }^{7}$ Manami Inoue, ${ }^{8,9}$ Norie Sawada, ${ }^{8}$ Shoichiro Tsugane, ${ }^{8}$ for the JPHC Study Group

To cite: Kato M, Noda M, Mizoue T, et al. Diagnosed diabetes and premature death among middle-aged Japanese: results from a large-scale population-based cohort study in Japan (JPHC study). BMJ Open 2015;5:e007736. doi:10.1136/bmjopen-2015007736

- Prepublication history and additional material is available. To view please visit the journal (http://dx.doi.org/ 10.1136/bmjopen-2015007736).

Received 20 January 2015 Revised 26 March 2015 Accepted 8 April 2015

CrossMark

For numbered affiliations see end of article.

Correspondence to Dr Mitsuhiko Noda; mnoda@hosp.ncgm.go.jp

\section{ABSTRACT}

Objective: To examine the association between diabetes and premature death for Japanese general people.

Design: Prospective cohort study.

Setting: The Japan Public Health Center-based prospective study (JPHC study), data collected between 1990 and 2010.

Population: A total of 46017 men and 53567 women, aged 40-69 years at the beginning of baseline survey.

Main outcome measures: Overall and cause specific mortality. Cox proportional hazards models were used to calculate the HRs of all cause and cause specific mortality associated with diabetes.

Results: The median follow-up period was 17.8 years. During the follow-up period, 8223 men and 4640 women have died. Diabetes was associated with increased risk of death (856 men and 345 women; HR $1.60,(95 \% \mathrm{Cl} 1.49$ to 1.71$)$ for men and 1.98 (95\% Cl 1.77 to 2.21) for women). As for the cause of death, diabetes was associated with increased risk of death by circulatory diseases (HR $1.76(95 \% \mathrm{Cl} 1.53$ to 2.02) for men and 2.49 (95\% $\mathrm{Cl} 2.06$ to 3.01 ) for women) while its association with the risk of cancer death was moderate (HR 1.25 (95\% Cl 1.11 to 1.42) for men and 1.04 (95\% Cl 0.82 to 1.32) for women). Diabetes was also associated with increased risk of death for 'noncancer, non-circulatory system disease' (HR 1.91 (95\% Cl 1.71 to 2.14) for men and $2.67(95 \% \mathrm{Cl} 2.25$ to 3.17) for women).

Conclusions: Diabetes was associated with increased risk of death, especially the risk of death by circulatory diseases.

\section{INTRODUCTION}

Today, Japanese people, especially Japanese women, are one of the people who live longest in the world. ${ }^{1}$ On the other hand, the prevalence of type 2 diabetes has increased over the past few decades in Japan and the

\section{Strengths and limitations of this study}

- A large scale population-based prospective study, the study population was defined as all registered Japanese inhabitants in the 11 public health centre areas, was conducted.

- In Japan, the registration of deaths is required by the Family Registration Law and is believed to be complete.

- The assessment of diabetes mellitus was based on a self-report. Although the sensitivity and specificity of diagnosed diabetes were reported to be high, the assessment of diabetes by selfreport is most likely an underestimate.

- The association between mortality and glycaemia was not examined because data about glycaemia were not available for the entire population.

total number of patients with diabetes is estimated to have risen from 7.4 million in 2002 to 9.5 million in $2012 .^{2}$ Diabetes is an important cause of mortality and morbidity and there are many literatures concerning the association between diabetes and mortality. However, most of these literatures were focused on the Western people and the impact of diabetes on premature death among Japanese people was not well examined. Several genetic and environmental differences as well as causes of death between Japanese and Western people exist and in the present study we examined the association between diagnosed diabetes and premature death for Japanese general people in a large scale population-based cohort study.

\section{METHODS}

The Japan Public Health Centre-based prospective study (JPHC study) consists of two 
cohort, Cohort I and Cohort II that comprise five and six prefectural public health centre areas, respectively. The JPHC Study group members are listed in online supplementary appendix. The study population was defined as all registered Japanese inhabitants in the 11 public health centre areas, aged 40-69 years at the beginning of each baseline survey, that is, in 1990 for Cohort I and in 1993 for Cohort II. Details of the study design have been described elsewhere. ${ }^{3}$ The study protocol was approved by the institutional review board of the National Cancer Centre.

Initially, 140420 participants were identified as the study population. Participants with non-Japanese nationality, duplicate enrolment, late report of emigration occurring before the start of follow-up or ineligibility because of incorrect birth date $(n=260)$ were excluded.

\section{Questionnaire}

At the baseline survey, each participant completed a selfadministered questionnaire that included questions about various lifestyle factors; such as medical history of major diseases, smoking and alcohol drinking status, height and weight and leisure-time physical activity. A similar survey was conducted at 5 and 10 years after the baseline survey.

At baseline, a total of 113402 participants responded to the questionnaire (response rate $80.9 \%$ ). Participants whose follow-up period was not determined were excluded from further analysis $(n=90)$. Participants with any of the following conditions at baseline: cardiovascular disease, chronic liver disease, kidney disease and any type of cancer, were also excluded $(n=8049)$. Participants who had missing baseline data for any of the exposure parameters described below (in Statistical Analysis; $n=5049$ ) or participants with a body mass index (calculated as weight in kilograms divided by the square of height in metres) of less than 14 or more than 40 $(n=1363)$ were also excluded, because body mass index less than 14 or more than 40 in Japanese implies potentially unreliable data. After the above exclusions, the remaining cohort consisted of 99584 participants (46 017 men and 53567 women).

\section{Assessment of diabetes}

We defined the participant as having diagnosed diabetes if he or she marked on 'diabetes mellitus' to the question 'Has a doctor ever told you that you have any of the following diseases?' or on 'anti-diabetic drug' to the question 'Do you take any of the following drugs?' The sensitivity and specificity of diagnosed diabetes was reported as $82.9 \%$ and $99.7 \%$, respectively. ${ }^{4}$ The questionnaire did not distinguish type 1 and type 2 diabetes. However, the participants of the present study were Japanese inhabitants aged 40-69 years and we believe that most of the participants with diagnosed diabetes had type 2 diabetes.

\section{Follow-up}

Participants were followed from the baseline survey up to 31 December 2010. All death certificates were forwarded centrally to the Ministry of Health, Welfare and Labor and coded for the National Vital Statistics. In Japan, the registration of deaths is required by the Family Registration Law and is believed to be complete. The underlying cause of death was determined by death certificates and was coded according to the 10th revision of the International Classification of Disease (ICD-10). Until 1995, the cause of death was determined according to the criteria of the ICD-9 and from 1995, the codes were translated into the corresponding ICD-10 codes.

\section{Statistical analysis}

Person-years of follow-up were counted from the date of the baseline survey until one of the following end points: the date of emigration from Japan, the date of death or the end of the study period (31 December 2010), whichever comes first. Age-standardised mortality rate was calculated by direct method using 5-year age-specific mortality rate and the total population (participants with and without diabetes) as standard. The association between diabetes and premature death was estimated as HRs using Cox's proportional hazards model with age as the time scale. ${ }^{5}$ We adjusted potential confounding factors: body mass index (categorised as 14-18.4, 18.5-24.9, 25-29.9 and 30-40), alcohol intake (categorised by weekly ethanol intake as non-drinker, 1-149 g/week, 150-299 g/week, 300-449 g/week and $\geq 450 \mathrm{~g}$ /week for men and the last two categories were combined into a category $\geq 300 \mathrm{~g}$ /week for women), smoking status (categorised as never smoker, past smoker, current smoker at $<20$ and $\geq 20$ cigarettes per day), leisure-time physical activity (dichotomised as participate in sports at least once a week or not) and history of hypertension. The public health center areas were included in the analysis as strata. Effect of birth cohort was also examined by including birth cohort (birth year of 1920-1929, 1930-1939 and 1940-). Difference of the association between diabetes and mortality by diagnosed period was also examined by including information about diagnosis of diabetes at 5 and 10-year survey for participants who responded to 5 and/or 10-year survey, that is, participants were classified into four groups according to the period of diagnosis of diabetes: diagnosed before baseline, diagnosed between baseline and 5 -year survey, diagnosed between 5 and 10-year survey, never diagnosed. Person-years of follow-up of participants diagnosed between baseline and 5-year survey and diagnosed between 5 and 10-year survey were counted from 5 and 10 years after the baseline survey, respectively.

HRs were calculated for death from all cause, circulatory system diseases (ICD-10, I00-I99), all cancer (ICD-10, C00-C97) and site-specific cancer if there were five or more cases in participants with diabetes. Deaths 
from other than circulatory system disease or cancer were grouped as 'non-cancer, non-circulatory system disease' and the HR for this group was also calculated. The proportional hazards assumption was checked graphically and by using Schoenfeld residuals.

All analyses were performed separately for men and women.

\section{RESULTS}

The median follow-up period was 17.8 years both for men and women. During the follow-up period, 8223 men and 4640 women have died. The baseline characteristics of the study participants are shown in table 1. At baseline, $6 \%$ of men and $2.8 \%$ of women had diagnosed diabetes. Among men, age, proportion of participants with leisuretime physical activity and history of hypertension were higher among participants with diabetes. Among women, age, the body mass index, proportion of participants with leisure-time physical activity and history of hypertension were higher among participants with diabetes. Besides these factors, medication about hypercholesterolaemia was higher among participants with diabetes $3.5 \%$ among men with diabetes and $1.2 \%$ among men without diabetes for and $5.5 \%$ among women with diabetes and $1.9 \%$ among women without diabetes).

Among men without diabetes, 1744 participants died from circulatory system disease, 3093 participants died from cancer and 2530 participants died from other causes, while among men with diabetes, these numbers were 230, 283 and 343, respectively. Among women without diabetes, 1084 participants died from circulatory system disease, 1841 participants died from cancer and 1370 participants died from other causes, while among women with diabetes, these numbers were 123,71 and 151 , respectively.
HRs for major causes of death were shown in table 2. As shown in table 2, diabetes was associated with increased risk of death both for men and women. The HR was high for circulatory system disease (ischaemic heart disease and cerebrovascular disease) among men and especially high for ischaemic heart disease and cerebral infarction among women. The association between diabetes and the risk of death from cancer was moderate and the HRs were not high except some types of cancer (liver cancer both among men and women and pancreas, kidney and bladder cancer among men), while death from 'multiple myeloma and malignant plasma cell neoplasms' in men and 'malignant neoplasm of breast' in women was markedly lower among participants with diabetes (46/0 cases for multiple myeloma and 135/1 cases for neoplasm of breast). Diabetes was also associated with increased risk of death for 'non-cancer, non-circulatory system disease'. These results were almost unchanged when the deaths during the first 5 years were excluded. The major causes of death for 'non-cancer, noncirculatory system disease' among participants with diabetes were 'unspecified diabetes mellitus' (E14) (men $17.8 \%$, women $22.5 \%$ ), 'pneumonia, organism unspecified' (J18) (men 13.7\%, women 13.9\%) and 'unknown causes' (men $6.4 \%$, women $10.6 \%$ ).

The HR of diabetes on mortality was larger among participants with diabetes diagnosed before baseline than among participants diagnosed after baseline (table 3). Differences of HRs between participants diagnosed between baseline and 5-year survey and participants diagnosed between 5 and 10-year survey were not clear.

No significant interaction was observed between adjustment factors and the results were essentially unchanged by including the effect of birth cohort. Further adjustment for medication for hypercholesterolaemia had little impact on our results. We found no

Table 1 Baseline characteristics according to diagnosed diabetes

\begin{tabular}{|c|c|c|c|c|c|c|c|c|}
\hline \multirow[b]{3}{*}{ Age } & \multicolumn{4}{|c|}{ Men $(n=46017)$} & \multicolumn{4}{|c|}{ Women ( $n=53567)$} \\
\hline & \multicolumn{2}{|c|}{$\overline{D M}(-)(n=43256)$} & \multicolumn{2}{|c|}{$D M(+)(n=2761)$} & \multicolumn{2}{|c|}{$\overline{D M}(-)(n=52042)$} & \multicolumn{2}{|c|}{$\mathrm{DM}(+)(\mathrm{n}=1525)$} \\
\hline & 50 & $(44-56)$ & 53 & $(49-59)$ & 50 & $(44-57)$ & 56 & $(50-62)$ \\
\hline BMI & 23.5 & $(2.8)$ & 23.7 & (3.0) & 23.3 & (3.1) & 24.4 & (3.6) \\
\hline \multicolumn{9}{|l|}{ Smoking } \\
\hline Never & 10175 & $(23.5)$ & 577 & (20.9) & 47347 & $(91.0)$ & 1363 & $(89.4)$ \\
\hline Past & 10106 & $(23.4)$ & 713 & (25.8) & 942 & $(1.8)$ & 45 & $(3.0)$ \\
\hline Current (<20 cigarettes/day) & 5913 & $(13.7)$ & 398 & (14.4) & 2422 & $(4.7)$ & 64 & $(4.2)$ \\
\hline Current ( $\geq 20$ cigarettes/day) & 17062 & $(39.4)$ & 1073 & (38.9) & 1331 & $(2.6)$ & 53 & (3.5) \\
\hline \multicolumn{9}{|l|}{ Alcohol } \\
\hline Non-drinker & 13248 & $(30.6)$ & 944 & (34.2) & 44844 & $(86.2)$ & 1386 & $(90.9)$ \\
\hline $1-150 \mathrm{~g} /$ week & 9667 & $(22.4)$ & 575 & $(20.8)$ & 5609 & $(10.8)$ & 103 & $(6.8)$ \\
\hline 150-300 g/week & 9032 & $(20.9)$ & 511 & $(18.5)$ & 996 & $(1.9)$ & 16 & $(1.0)$ \\
\hline $300-450 \mathrm{~g} /$ week ( $\geq 300$ week for women) & 5325 & (12.3) & 273 & $(9.9)$ & 593 & $(1.1)$ & 20 & (1.3) \\
\hline$\geq 450 \mathrm{~g} /$ week & 5984 & (13.8) & 458 & $(16.6)$ & & & & \\
\hline Physical activity (active) & 8188 & (18.9) & 682 & (24.7) & 9629 & (18.5) & 381 & $(25.0)$ \\
\hline Hypertension (+) & 7218 & (16.7) & 795 & (28.8) & 8145 & (15.7) & 545 & (35.7) \\
\hline
\end{tabular}




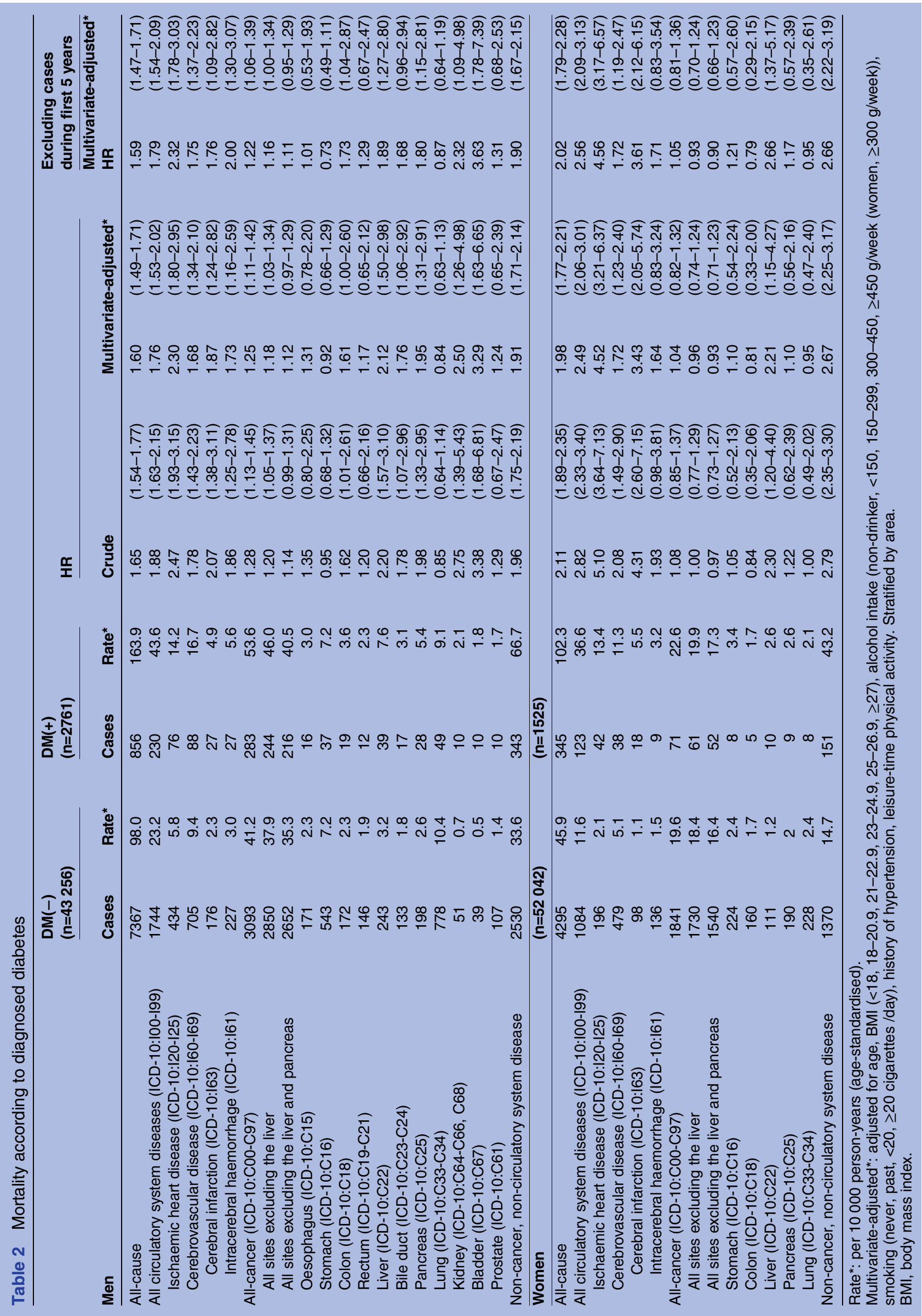


Table 3 Difference of the association between diabetes and mortality by diagnosed period (All-cause mortality)

\begin{tabular}{|c|c|c|c|c|c|c|c|c|}
\hline \multirow[b]{2}{*}{ Diagnosed period } & \multicolumn{4}{|l|}{ Men } & \multicolumn{4}{|c|}{ Women } \\
\hline & $\overline{\mathbf{n}}$ & Cases & Adju & ed $\mathrm{HR}^{\star}$ & $\overline{\mathbf{n}}$ & Cases & Adjus & ed $H^{*}$ \\
\hline Never & 41036 & 6975 & 1 & & 50555 & 4106 & 1 & \\
\hline Before baseline & 2761 & 856 & 1.59 & $(1.48-1.71)$ & 1525 & 345 & 2.00 & $(1.79-2.23)$ \\
\hline Between baseline and 5-year survey & 1341 & 254 & 1.20 & $(1.05-1.36)$ & 861 & 123 & 1.55 & $(1.29-1.86)$ \\
\hline Between 5 and 10-year survey & 879 & 138 & 1.22 & $(1.03-1.44)$ & 626 & 66 & 1.45 & $(1.14-1.86)$ \\
\hline
\end{tabular}

Adjusted $\mathrm{HR}^{*}$ : adjusted for $\mathrm{BMI}(<18,18-20.9,21-22.9,23-24.9,25-26.9, \geq 27)$, alcohol intake (non-drinker, $<150,150-299,300-450$, $\geq 450 \mathrm{~g} /$ week (women, $\geq 300 \mathrm{~g} /$ week)), smoking (never, past, $<20, \geq 20$ cigarettes /day), history of hypertension, leisure-time physical activity. Stratified by area.

BMI, body mass index.

violation of proportionality assumption. However, although it was not confirmed statistically, there was a tendency that the HR of diabetes for death decreased as age increased (figure 1).

\section{DISCUSSION}

In this population-based prospective study of middleaged Japanese, we observed the increased risk of death for participants with diabetes. As for the cause of death, diabetes was associated with increased risk of death by circulatory system diseases and 'non-cancer, noncirculatory system disease', while the association with the risk of death from cancer was moderate.

There are many literatures about diabetes and mortality and substantial numbers of these results were combined into the Emerging Risk Factors Collaboration (ERFC). ${ }^{6}$ In ERFC, the HRs among participants with diabetes compared with participants without diabetes were reported as 1.80 for all-cause mortality, 1.25 for death from cancer, 2.32 for death from vascular causes and 1.73 for death from other causes.

Results of another large prospective cohort study of one million US adults (CPS-II) was also published. ${ }^{7}$ In the study, relative risk of all-cause mortality was 1.73 for men and 1.90 for women and that of cancer death was 1.07 for men and 1.11 for women and that of cardiovascular system death was 1.92 for men and 2.09 for women.

Recently published meta-analysis also reported increased mortality among participants with diabetes

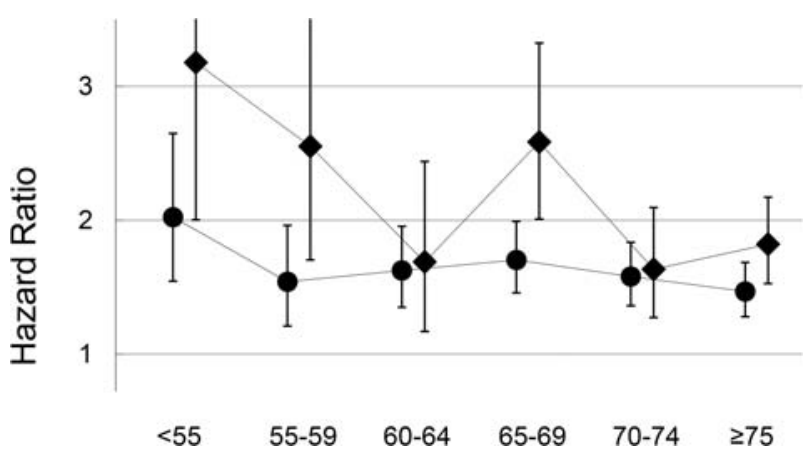

Figure 1 Changes of HR of diabetes according to age (all-cause mortality; Men, circle; Women, diamond). and the relative risk for all-cause mortality was 1.57 for men and 2.00 for women and that of cardiovascular mortality was $1.76 .^{8}$

Although the results were almost similar, there is a difference of major causes of death between our study and these studies. In the present study, $41 \%$ and $25 \%$ of all deaths were caused by cancer and circulatory system disease, respectively, while these numbers were $34 \%$ and $36 \%$ in the ERFC and $15 \%$ and $50 \%$ in the CPS-II, respectively. This tendency that Japanese die from cancer more than from circulatory system disease and that this is opposite for western people (although ERFC was a collation of over 100 prospective studies, about $90 \%$ of the participants were from North America or Europe), is also observed in the world statistics. ${ }^{9}$ As discussed above, diabetes was associated with increased risk of death by circulatory system disease more than death by cancer. This may seem as if the association between diabetes and mortality is stronger in a population among which the major cause of death was circulatory system disease, that is, the association is stronger among western people than Japanese. However, this is not true because 'non-cancer, non-circulatory system disease' plays an unignorable part of mortality.

Our results were also almost consistent with the Japanese large scale cohort study (Takayama study). ${ }^{10}$ The most remarkable difference between the Takayama study and the present study was the risk of death by coronary heart disease among women. In the Takayama study, the risk of death by coronary heart disease among women were lower in participants with diabetes than participants without diabetes (HR 0.49, 95\% CI 0.07 to 3.57). As shown in the wide CI, this difference may come from the very low number of cases (only two cases) of coronary heart disease death among women with diabetes. The collaborate study in $\mathrm{Asia}^{11}$ and meta-analysis including this collaborate study ${ }^{12}$ and its update $^{13}$ reported the increased risk of coronary heart disease among women with diabetes and our results were consistent with these reports. Our study revealed that the effect of diabetes on the risk of cardiovascular death was greater among women than among men. This is also consistent with the aforementioned metaanalysis. ${ }^{12}{ }^{13}$ Although several possible explanations, such as (1) a heavier burden of cardiovascular risk 
factors, (2) a major impact of some cardiovascular risk factors and/or diabetes per se on cardiovascular disease, (3) differences in the structure and function of heart and vessels and (4) disparities in medical treatment as well as gender differences in treatment response, are postulated, the underlying mechanism of this sex difference in the impact of diabetes on cardiovascular disease is not elucidated well. ${ }^{14}$

As for the death from cancer, our results were almost consistent with the report about the incidence of cancer in the same JPHC study. ${ }^{15}$ In the case of incidence, diabetes moderately increased the risk of all cancer and the risk was especially high for cancer of the liver, pancreas and kidney among men and for cancer of the stomach, liver and ovary among women. In the present study, a similar tendency was observed among men, however the number of death from cancer was small among women and the increased mortality risk associated with diabetes was observed only in liver cancer.

We found that the association between diabetes and mortality was stronger among participants diagnosed before baseline than among participants diagnosed after baseline. This result suggests that the effect of diabetes on mortality becomes stronger as duration of diabetes becomes longer.

We also found, although not confirmed statistically, that the HR of diabetes for death decreased as age increased. The similar phenomenon was observed in the ERFC. The reason is unclear. However, one possible explanation is that patients with diabetes who lived long managed their diabetes relatively well. Another possible explanation is that the patients with diabetes with older age included more recently developed diabetes because the risk of diabetes increases as age increases and, as stated above, the association between diabetes and mortality was relatively weaker in newly developed diabetes.

The strength of our study was the large number of participants. The number of participants was about 3.4 times that of the Takayama study. Another strength of the present study was that it was based on the general population in Japan. Although this study was conducted on participants who responded to the baseline questionnaire, we believe that the high-response rate $(80.9 \%)$ makes it possible to assess the association between diabetes and mortality in the general population. In addition, the age-specific mortality rates in the present study were similar to those of Japanese general population. For example, age-specific mortality rates (per 10000 person-years) in the present study in men were 15.5, 36.3, 83.0 and 224.0 for 40, 50, 60 and 70-years-old, respectively, and those of Japanese general population (Abridged Life Tables For Japan 2005) were 14.4, 35.8, 89.4 and 213.8. In women, the age-specific mortality rates (per 10000 person-years) in the present study were 5.3, 17.4, 33.2 and 87.2 for 40, 50, 60 and 70-years-old, respectively and those of Japanese general population were 7.5, 17.7, 36.6 and 89.3. No large discrepancies in mortality rates exist between our study and
Japanese general population and this may also support the representativeness of our cohort.

There are several methodological limitations in the present study. The assessment of diabetes mellitus was based on a self-report. Although the sensitivity (82.6\%) and specificity $(99.7 \%)$ of diagnosed diabetes were reported to be high, the proportion of participants with diabetes at baseline (6\% for men and $2.8 \%$ for women) was low compared with the estimates in the same period $(9.9-13.1 \%$ for men and $9.1-11.5 \%$ for women $) .{ }^{16}$ The assessment of diabetes by self-report, therefore, is most likely an underestimate and our results may have been distorted toward null by this misclassification. However, in the aforementioned meta-analysis, sensitivity analyses were performed and no difference was found in the ratio of the relative risks for diabetes between the method of diabetes diagnosis (self-report vs glucose measured). ${ }^{12}$ Previous studies have revealed the association between mortality and glycaemia in patients with diabetes ${ }^{17}$ and this association holds even in the nondiabetic range of glycaemia. ${ }^{7}{ }^{18}$ Since we have no data about glycaemia, we could not assess the association between mortality and glycaemia in the present study.

Despite these limitations, our present study revealed the association between diabetes and mortality in the Japanese general population. Recent increase in patients with diabetes will influence the longevity of Japanese people in the future and we believe that our study would provide useful information both for further research and treatment of diabetes.

\section{Author affiliations}

${ }^{1}$ Department of Diabetes Research, Diabetes Research Center, National Center for Global Health and Medicine, Tokyo, Japan

${ }^{2}$ Fiore Kenshin Clinic, Tokyo, Japan

${ }^{3}$ Department of Epidemiology and Prevention, Center for Clinical Sciences, National Center for Global Health and Medicine, Tokyo, Japan

${ }^{4}$ Department of Public Health, Tokyo Women's Medical University, Tokyo, Japan

${ }^{5}$ Division of Diabetes and Metabolism, Iwate Medical University School of Medicine, Morioka, Japan

${ }^{6}$ Department of Clinical Research, Center for Clinical Sciences, National Center for Global Health and Medicine, Tokyo, Japan

${ }^{7}$ Department of Public Health, Department of Social and Environmental Medicine, Osaka University, Graduate School of Medicine, Osaka-fu, Japan ${ }^{8}$ Epidemiology and Prevention Group, Research Center for Cancer Prevention and Screening, National Cancer Center, Tokyo, Japan

${ }^{9}$ Graduate School of Medicine, The University of Tokyo, Tokyo, Japan

Acknowledgements The authors are grateful to Dr Mayako Takemoto for her support in preparing the manuscript in English.

Contributors MK analysed data, drafted the manuscript, reviewed and edited the manuscript and contributed to discussion. MN and ST conducted, designed and supervised the study, and contributed to discussion. TM, AG, YT, YM, AN, HI, MI and NS reviewed the manuscript and contributed to discussion. MN is the guarantor. All authors read and approved the final manuscript.

Funding The present study was supported by National Cancer Center Research and Development Fund (23-A-31(toku) and 26-A-2) (since 2011) and a Grant-in-Aid for Cancer Research from the Ministry of Health, Labour and Welfare of Japan (from 1989 to 2010) and a Health Sciences Research Grant (Research on Comprehensive Research on Cardiovascular Diseases H19-016) from the Ministry of Health, Labour and Welfare of Japan. 
Competing interests $\mathrm{MI}$ is the beneficiary of a financial contribution from the AXA Research fund as a chair holder on the AXA Department of Health and Human Security, Graduate School of Medicine, The University of Tokyo.

Ethics approval National Cancer Center.

Provenance and peer review Not commissioned; externally peer reviewed.

Data sharing statement No additional data available.

Open Access This is an Open Access article distributed in accordance with the Creative Commons Attribution Non Commercial (CC BY-NC 4.0) license, which permits others to distribute, remix, adapt, build upon this work noncommercially, and license their derivative works on different terms, provided the original work is properly cited and the use is non-commercial. See: http:// creativecommons.org/licenses/by-nc/4.0/

\section{REFERENCES}

1. World Health Organization. World Health Statistics, 2011.

2. National Institute of Health and Nutrition. Outline of the National Health and Nutrition Survey Japan. 2007.

3. Tsugane S, Sobue T. Baseline survey of JPHC study-design and participation rate. Japan Public Health Center-based Prospective Study on Cancer and Cardiovascular Diseases. J Epidemiol 2001;11 (6 Suppl):S24-9.

4. Kato M, Noda M, Inoue M, et al. Psychological factors, coffee and risk of diabetes mellitus among middle-aged Japanese: a population-based prospective study in the JPHC study cohort. Endocr J 2009;56:459-68.

5. Korn EL, Graubard BI, Midthune D. Time-to-event analysis of longitudinal follow-up of a survey: choice of the time-scale. Am J Epidemiol 1997;145:72-80.

6. Emerging Risk Factors Collaboration, Seshasai SR, Kaptoge S, Thompson A, et al. Diabetes mellitus, fasting glucose, and risk of cause-specific death. N Engl J Med 2011;364:829-41.
7. Saydah S, Tao M, Imperatore G, et al. GHb level and subsequent mortality among adults in the U.S. Diabetes Care 2009;32:1440-6.

8. Nwaneri C, Cooper H, Bowen-Jones D. Mortality in type 2 diabetes mellitus: magnitude of the evidence from a systematic review and meta-analysis. Br J Diabetes Vasc Dis 2013;13:192-207.

9. Health statistics and health information systems: mortality data. http://www.who.int/healthinfo/statistics/mortality/en/index.html

10. Oba S, Nagata C, Nakamura K, et al. Self-reported diabetes mellitus and risk of mortality from all causes, cardiovascular disease, and cancer in Takayama: a population-based prospective cohort study in Japan. J Epidemiol 2008;18:197-203.

11. Woodward M, Zhang X, Barzi F, et al. The effects of diabetes on the risks of major cardiovascular diseases and death in the Asia-Pacific region. Diabetes Care 2003;26:360-6.

12. Huxley R, Barzi F, Woodward M. Excess risk of fatal coronary heart disease associated with diabetes in men and women: meta-analysis of 37 prospective cohort studies. BMJ 2006;332:73-8.

13. Peters SE, Huxley R, Woodward M. Diabetes as risk factor for incident coronary heart disease in women compared with men: a systematic review and meta-analysis of 64 cohorts including 858,507 individuals and 28,203 coronary events. Diabetologia 2014;57:1542-51.

14. Rivellese AA, Riccardi G, Vaccaro O. Cardiovascular risk in women with diabetes. Nutr Metab Cardiovasc Dis 2010;20:474-80.

15. Inoue M, Iwasaki M, Otani T, et al. Diabetes mellitus and the risk of cancer: results from a large-scale population-based cohort study in Japan. Arch Intern Med 2006;166:1871-7.

16. Kuzuya T. Prevalence of diabetes mellitus in Japan compiled from literature. Diabetes Res Clin Pract 1994;24(Suppl):S15-21.

17. Stratton IM, Adler AI, Neil HA, et al. Association of glycaemia with macrovascular and microvascular complications of type 2 diabetes (UKPDS 35): prospective observational study. BMJ 2000;321: 405-12.

18. Khaw KT, Wareham N, Luben R, et al. Glycated haemoglobin, diabetes, and mortality in men in Norfolk cohort of European Prospective Investigation of Cancer and Nutrition (EPIC-Norfolk). BMJ 2001;322:15-18. 